OPEN ACCESS

Edited by:

Sarah K. Tasian,

Children's Hospital of Philadelphia,

United States

Reviewed by:

Liora Schultz,

Stanford University, United States

*Correspondence:

Christine Moore Smith

christine.m.smith.2@vumc.org

Specialty section:

This article was submitted to

Pediatric Oncology,

a section of the journal

Frontiers in Oncology

Received: 15 January 2022 Accepted: 04 February 2022

Published: 25 February 2022

Citation:

Smith CM and Friedman DL (2022) Advances in Hodgkin Lymphoma: Including the Patient's Voice.

Front. Oncol. 12:855725.

doi: 10.3389/fonc.2022.855725

\section{Advances in Hodgkin Lymphoma: Including the Patient's Voice}

\author{
Christine Moore Smith ${ }^{1,2 *}$ and Debra L. Friedman ${ }^{1,2}$ \\ 1 Division of Pediatric Hematology/Oncology, Vanderbilt University Medical Center, Nashville, TN, United States, \\ 2 Vanderbilt-Ingram Cancer Center, Vanderbilt University Medical Center, Nashville, TN, United States
}

Since the initial treatment with radiation therapy in the 1950s, the treatment of Hodgkin lymphoma has continued to evolve, balancing cure and toxicity. This approach has resulted in low rates of relapse and death and fewer short and late toxicities from the treatments used in pursuit of cure. To achieve this balance, the field has continued to progress into an exciting era where the advent of more targeted therapies such as brentuximab vedotin, immunotherapies such as PD-1 inhibitors, and chimeric antigen receptor T-cells (CAR-T) targeted at CD30 are changing the landscape. As in the past, cooperative group and international collaborations are key to continuing to drive the science forward. Increased focus on patient-reported outcomes can further contribute to the goal of improved outcomes by examining the impact on the individual patient in the acute phase of therapy and on long-term implications for survivors. The goals of this review are to summarize recent and current clinical trials including reduction or elimination of radiation, immunotherapies and biologically-targeted agents, and discuss the use of patient-reported outcomes to help discern directions for new therapeutic regimens and more individualized evaluation of the balance of cure and toxicity.

Keywords: Hodgkin, lymphoma, patient-reported outcomes, immunotherapy, survivorship, targeted therapy, Hodgkin lymphoma (HL)

\section{INTRODUCTION}

Hodgkin Lymphoma (HL) is a malignant lymphoma with an impact spanning both the pediatric and adult populations. Cases occur in a bimodal distribution with peak in the adolescent and young adult (AYA) population, with varying definitions, but commonly considered to encompass the ages of 15-39 years (1-3). Within pediatrics, the incidence of $\mathrm{HL}$ is 12.2 per million for children under age 20 , but 32 per million in ages 15-19, and highest between $20-24$ years at 45 per million $(4,5)$.

With current treatment options, HL has a high cure rate. After the advent of successful treatment with radiotherapy (RT) and then subsequently chemotherapy, death rates from HL have declined since 1975, with an additional impressive decrease of 4\% per year from 2008 to 2017. Recent data highlight excellent overall survival (OS) of $87 \%$ at 5 years across the age span and $95 \%$ for pediatric patients $(1,6)$. The failure rate of first-line therapies has similarly declined with $90 \%$ event free survival (EFS) in early stage disease and $80-85 \%$ EFS in advanced staged disease (7). Clinical trials have been essential in contributing to these improvements. With such high survival rates, focus over the last three decades has shifted to reducing both the acute and long-term effects of treatment while maintaining long-term EFS and OS. This focus is important as treatment of relapsed/refractory 
disease requires additional exposure to toxicity through salvage regimens, RT, and potentially high dose therapy with autologous hematopoietic cell transplant (HDT/AHCT) (6).

Varied multimodal approaches to achieve these goals have been studied in clinical trials, albeit without clear consensus on the best approach. As we move forward with efficacious regimens, we continue to learn how to best incorporate, prioritize, and sequence the use of newer agents. This can be enhanced, in part, by incorporating patient-reported outcomes (PROs). This is an exciting step toward understanding the patient-level impact of regimens on EFS, OS, and tolerability of acute and long-term effects of treatment.

\section{RISK ADAPTED THERAPY IN FRONTLINE CLINICAL TRIALS}

Clinical trials in the last two decades have explored effective multiagent chemotherapy regimens for response-based risk adaptation. For patients with early responses to chemotherapy, most regimens balance curative goals with late toxicity by omitting or reducing $\mathrm{RT}$. Reduction in radiation doses and fields spare normal tissues and are anticipated to decrease radiation-associated adverse long-term health effects (8). The chemotherapy backbones decreased alkylators and anthracyclines to minimize long-term adverse effects of these agents including fertility issues, secondary malignant neoplasms, and cardiotoxicity. While these have been central goals, approaches have varied somewhat in different pediatric collaborative groups including the Children's Oncology Group (COG), the St Jude-Stanford-Dana Farber Consortium, the German Paediatric Haematology-Oncology Group, and the European Network for Paediatric Hodgkin Lymphoma (EuroNet-PHL), among others. With the advent of the National Cancer Institute sponsored National Clinical Trials Network (NCTN), there is also the opportunity for further collaboration between pediatric and adult cooperative groups in the United States.

Table 1 reviews some of these more recent studies from pediatric and adult cooperative groups (9-16). The next generation of investigation builds upon these studies with incorporation of more biologically-targeted approaches.

\section{ADVANCES IN TREATMENT WITH BRENTUXIMAB VEDOTIN}

Brentuximab vedotin (Bv) is an antibody-drug conjugate that targets delivery of monomethyl auristain E to cells expressing CD30 such as the Reed Sternberg cells in classical HL.

Initially studied in the relapsed/refractory setting in ages $\geq 12$ including adults, a study of $\mathrm{Bv}$ monotherapy showed efficacy with CR in $38 \%$ and some durable remissions while overall being well-tolerated $(17,18)$.

Pediatric and AYA regimens have combined $\mathrm{Bv}$ with traditional cytotoxic chemotherapy in the relapsed/refractory disease setting. For patients $\leq 30$, COG AHOD1221 evaluated $\mathrm{Bv}$ with gemcitabine and reported $67 \%$ of patients achieved CR after 4 cycles when including patients meeting modern Deauville score criteria. Ad hoc analysis showed 1-year OS of 95\% (19). Bv has also been studied with bendamustine in several trials including the pediatric and AYA populations with CR rates of $66-79 \%$ and 2 and 3 -year progression free survival (PFS) of 62.6$69.8 \%(20-23)$.

In pediatrics, several studies have also incorporated $\mathrm{Bv}$ in frontline treatment. A single-arm trial led by the St. JudeStanford-Dana-Farber Consortium for ages $\leq 18$ evaluated the safety and efficacy of $\mathrm{Bv}$ for high-risk patients in a backbone of A-EPA/CAPDac (Bv, etoposide, prednisone, doxorubicin, cyclophosphamide, dacarbazine). Results included 3-year EFS of $97.4 \%$, and $35 \%$ of patients were early responders avoiding need for RT. The study highlights tolerability of Bv and effectiveness of residual node radiation (24). AHOD1331, a trial by the COG for ages 2-21, completed accrual of highrisk patients treated with a backbone of ABVE-PC (doxorubicine, bleomycin, vincristine, prednisone, cyclophosphamide) compared with $\mathrm{Bv}$ substitution for bleomycin. Need for involved site RT was determined by PET response. Data release and analyses are expected in the near future (25).

For frontline trials in adult patients, the ECHELON-1 trial compared the standard of ABVD (doxorubicin, bleomycin, vinblastine, dacarbazine) with A-AVD (Bv, doxorubicin, vinblastine, dacarbazine) for advanced stage disease in patients ages $\geq 18$. The 3 -year PFS was superior for A-AVD versus ABVD $(83.1 \%$ versus $76 \%)(26,27)$.

The most notable dose limiting toxicity of $\mathrm{Bv}$ is neuropathy which is reported subjectively by patients and has been shown to be tolerable and reversible in most trials. Reliance on the patient experience for toxicity reporting exemplifies how standardized PROs can help measure tolerability to determine which regimens best balance efficacy and toxicity $(24,27,28)$.

\section{ADVANCES IN TREATMENT WITH IMMUNE CHECKPOINT INHIBITORS}

HL cells have overexpression of programmed death-1 (PD-1) ligands 1 and 2 due to alterations in the 9p24.1 locus, and PD-L1 is also expressed in tumor associated macrophages making immune checkpoint inhibitors (ICIs), specifically anti-PD-1 monoclonal antibodies, promising agents for investigation in HL (29-31). Cytotoxic T lymphocyte-associated protein 4 (CTLA-4) blockade is an alternate approach which activates peritumoral $\mathrm{T}$ cells to overcome $\mathrm{T}$ cell exhaustion in the tumor microenvironment. Ipilimumab is a monoclonal antibody targeting CTLA- 4 currently being evaluated both alone and in combination as it has shown synergy with nivolumab in other cancers such as melanoma $(32,33)$. A number of studies first conducted among adults have demonstrated promising results in $\mathrm{HL}$, and pediatric trials are now underway to ascertain if similar results can be attained in younger patients. 
TABLE 1 | Recent Cooperative Group Studies.

\begin{tabular}{|c|c|c|c|}
\hline $\begin{array}{l}\text { Cooperative } \\
\text { Group/Study }\end{array}$ & Goals & $\begin{array}{c}\text { Chemotherapy and } \\
\text { Radiotherapy }\end{array}$ & Outcomes and Notes \\
\hline $\begin{array}{l}\text { COG } \\
\text { AHOD0031 } \\
\text { (9) }\end{array}$ & $\begin{array}{l}\text { Response-based risk-adaptation for } \\
\text { reduction of RT; evaluate intensification of } \\
\text { chemotherapy for intermediate-risk patient }\end{array}$ & $\begin{array}{l}\text { ABVE-PC +/- DECA } \\
\text { IFRT to } 21 \text { Gy based on disease at } \\
\text { presentation if not in CR at early response } \\
\text { assessment }\end{array}$ & $\begin{array}{l}\text { EFS }=85 \% \text {; OS }=97.8 \% \\
\text { For early responders, IFRT did not significantly change } \\
\text { EFS. Chemotherapy intensification to DECA versus no } \\
\text { DECA did not significantly change EFS for slow } \\
\text { responders (9). }\end{array}$ \\
\hline
\end{tabular}

\begin{tabular}{ll}
\hline COG & Response-based risk-adaptation for \\
AHOD0431 & reduction of chemotherapy and RT for low- \\
(10) & risk patients with an integrated \\
& chemotherapy plus RT salvage regimen
\end{tabular}

\section{Frontline: AVPC}

Relapse: IV + DECA

IFRT to 21 Gy based on disease at presentation if not in $\mathrm{CR}$ at early response assessment or at relapse

\begin{tabular}{ll}
\hline COG & $\begin{array}{l}\text { Response-based risk-adaptation for } \\
\text { AHOD0831 } \\
\text { reduction of cumulative alkylators and RT in } \\
\text { high-risk patients }\end{array}$ \\
\hline EuroNet- & $\begin{array}{l}\text { Comparison of consolidation regimens and } \\
\text { PHL }\end{array}$ \\
reduction of RT; results published for \\
\end{tabular}

ABVE-PC +/- IV

IFRT to 21 Gy to initial bulky disease and sites of slow response

OEPA + COPP vs COPDAC

RT to 19.8 Gy at all initially involved tumor sites for patients with inadequate response to chemotherapy alone; additional 10 Gy boost to bulky sites or slow response

\begin{tabular}{ll}
\hline EuroNet- & Evaluate intensification of chemotherapy \\
PHL & from COPDAC-28 to DECOPDAC-21 and \\
C2 (13) & reduce use of RT by targeting FDG-avid \\
& sites of disease at end of chemotherapy
\end{tabular}

OEPA +/- COPDAC-28 vs DECOPDAC-21

in certain cases

Randomization depending on risk group and early and late response assessments; dose ranges from 19.8 Gy to 30 Gy

$\begin{array}{ll}\text { ECOG } & \text { Compared chemotherapy regimens for } \\ \text { E2496 (14) } & \text { superiority of Stanford V over ABVD }\end{array}$

ABVD vs Stanford V

RT to 36 Gy for all bulky mediastinal adenopathy; RT on Stanford V arm to 36 Gy for lesions $>5 \mathrm{~cm}$ or macroscopic splenic disease

\begin{tabular}{lll}
\hline SWOG & Evaluate intensification of therapy if PET2 & ABVD +/- eBEACOPP \\
s0816 & positive & None \\
$(15,16)$ & &
\end{tabular}

\section{If $\mathrm{CR}$ on FDG-PET scan (PET) after 1 cycle of}

chemotherapy, the 4-year EFS was $88.2 \%$ versus $68.5 \%$. Patients with low stage mixed cellularity histology had an excellent EFS of $95.2 \%$

5-year EFS (all patients) = 79.1\%; Rapid early response $\mathrm{EFS}=83.5 \%$; Slow early response EFS $=73.2 \%$. EFS was below the prespecified target for the trial.

$49 \%$ of intermediate and $35 \%$ of high-risk with adequate response to chemotherapy and did not have subsequent RT with 5-year EFS $=90.1 \%$. Patients on the COPP arm had EFS of $89.9 \%$ and COPDAC $86.1 \%$.

Results not yet available. Notably moved toward the more modern definition of Deauville positivity of 4 and 5 , which will increase the number of patients eligible for elimination of RT.

No significant difference in response rate or in failure-free survival. Toxicity was reported to be similar between the two arms. The authors concluded that ABVD should remain the standard of care.

PET2 was negative for $82 \%$ of patients; 5 -year PFS = $76 \%$ for PET2 negative versus 66\% for PET2 positive.

Recent collaborative group clinical trials with response-based risk-adjusted chemotherapy and radiotherapy (RT). ABVE-PC: doxorubicin, bleomycin, vincristine, etoposide, prednisone, cyclophosphamide; DECA: dexamethasone, etoposide, cisplatin, cytarabine; IV: vinorelbine, ifosfamide; AVPC: doxorubicin, vincristine, prednisone, cyclophosphamide; OEPA: vincristine, etoposide, prednisone, doxorubicin; COPP: cyclophosphamide, vincristine, prednisone, procarbazine; COPDAC(-28): cyclophosphamide, vincristine, prednisone, dacarbazine; DECOPDAC-21: 21 day cycle of COPDAC; ABVD: doxorubicin, bleomycin, vinblastine, dacarbazine; Stanford V: doxorubicin, vinblastine, chlormethine, vincristine, bleomycin, etoposide, prednisone; IFRT: involved field radiotherapy.

CheckMate 205 evaluated nivolumab, a PD-1 inhibitor, in several cohorts of patients ages $\geq 18$, including relapsed/ refractory disease as well as upfront with nivolumab monotherapy followed by a combination regimen of N-AVD (nivolumab, doxorubicin, vinblastine, dacarbazine). There were good overall response rates (ORR) of $71 \%$ in the relapsed/ refractory cohort and $21 \%$ CR. Patients in CR had a longer median PFS (37 months) versus partial response (15 months). Upfront responses were higher with $67 \%$ achieving $\mathrm{CR}$ and, at time of the report, $92 \%$ PFS at a median of 9 months $(31,34-36)$.

KEYNOTE-087 evaluated pembrolizumab, a PD-1 inhibitor, in patients ages $\geq 18$ with relapsed/refractory disease in multiple cohorts, with good ORR of $71.9 \%$ though only $27.6 \%$ achieved CR with a median duration of response of 16.5 months (37).

To evaluate combinations of ICIs, E4412, led by the ECOGACRIN Cancer Research Group, evaluated combinations of Bv with nivolumab, $\mathrm{Bv}$ with ipilimumab, or triplet therapy in ages $\geq 18$ and has now expanded recruitment through the COG to include children and adolescents $\geq 12$ with relapsed/refractory disease (38). The ipilimumab group showed $76 \%$ ORR, nivolumab $89 \%$, and triplet therapy $82 \%$; the PFS and OS are not yet fully reported. The triplet therapy had more adverse events than two agent combinations in the adult population, but this will remain to be seen in pediatrics and could inform which regimens are best to pursue in future trials $(39,40)$.

In pediatrics, the COG evaluated nivolumab as a single agent in relapsed or refractory solid tumors and lymphomas in ADVL1412 which showed 3 of 10 patients with HL, all of whom had PD-L1 expression, had responses (41).

Frontline collaborative studies now exist between pediatric and adult study groups (COG, EuroNET-PHL, NCTN, SWOG Cancer Research Network, Alliance for Clinical Trials in Oncology, ECOG-ACRIN, and NRG Oncology) to evaluate PD-1 inhibitors with chemotherapy in combined adult-pediatric populations. While open for all stages for adult patients, pediatric patients ages 3-25 can enroll on the low-risk arm of KEYNOTE-667, also known in COG as AHOD1822, evaluating the addition of pembrolizumab for patients with less than CR after 2 cycles of 
ABVD (42). Led by SWOG, S1826 randomizes patients ages $\geq 12$ with higher risk disease between A-AVD versus N-AVD (43).

As these agents are now being more widely used in both upfront and relapsed/refractory regimens, there is evidence that rechallenge with targeted agents such as $\mathrm{Bv}$ or ICIs can be efficacious and tolerable even if there has been progressive disease or prior doselimiting toxicity related to the agent $(39,44,45)$.

\section{ADDITIONAL ADVANCES IN TREATMENT OF RELAPSED AND REFRACTORY DISEASE}

Refractory HL occurs in up to $5-10 \%$ of cases and $10-30 \%$ of patients will experience relapse, though these numbers are lower in pediatric only trials (39). Some of the factors affecting risk stratification include time to relapse, primary refractory disease, heavy pretreatment with radiation and/or chemotherapy, extranodal disease, higher stage/risk group, anemia, and B symptoms at relapse (39). Historically, combination chemotherapy regimens were the salvage approach for relapsed and refractory disease. More information about these chemotherapy regimens can be found in reviews by Voorhees and Beaven and in the National Comprehensive Cancer Network (NCCN) Clinical Practice Guidelines for Hodgkin lymphoma and Pediatric Hodgkin lymphoma $(39,46,47)$. Disease that has previously been chemotherapy responsive is a positive prognostic factor for success of such regimens for recurrent disease. However, for those with chemotherapy refractory disease, salvage regimens utilizing chemotherapy alone are likely less effective. Thus, biologically-targeted agents such $\mathrm{Bv}$, ICIs, chimeric antigen receptor T-cells (CAR T), and molecular targets are exciting options for those with recurrent or refractory disease (39).

Traditionally, HDT/AHCT has been considered the standard of care for most relapsed/refractory HL. However, this approach may be challenged somewhat as new biologicallytargeted agents are incorporated. The EuroNet-PHL published recommendations regarding who may benefit from HDT/ AHCT versus chemotherapy/immunotherapy and/or RT alone. They propose a risk stratification based on time to relapse (primary refractory/progression, early relapse 3-12 months, or late relapse after 12 months), significant prior treatment, stage at relapse, and response to salvage therapy (7). Complete metabolic response (CMR) is also a key component for prognosis with HDT/AHCT, though this can be complicated by the use of PD-1 inhibitors that can cause FDG-avidity leading to difficulty interpreting response on PET (44). A review by Harker-Murray highlighted similar risk factors to determine the utility of HDT/AHCT (48).

A phase II study for ages $\geq 18$ evaluated a combination of pembrolizumab with gemcitabine, vinorelbine, and liposomal doxorubicin. The regimen was efficacious in achieving CR in 95\% with few toxicities allowing continuation to HDT/AHCT and maintaining remission at a median of 13.5 months (49).

In studies with combined pediatric and adult patients, targeted agents are being combined with chemotherapy. The
COG study AHOD1721 evaluated Bv with nivolumab for ages 5-30. This regimen was well-tolerated with 59\% in CMR after 4 cycles. For those not in CMR, 2 cycles of $\mathrm{Bv}$ and bendamustine were added leading to $88 \%$ of patients achieving CMR prior to consolidative therapy with HDT/AHCT off study $(50,51)$.

Continued maintenance therapies with or without HDT/AHCT with ICIs and $\mathrm{Bv}$ are another strategy with encouraging efficacy data $(28,39,52,53)$. In adults, the AETHERA trial evaluated the use of $\mathrm{Bv}$ in patients ages $\geq 18$ as maintenance therapy following HDT/AHCT and demonstrated improvement in PFS $(52,53)$. A trial of pembrolizumab post-HDT/AHCT in patients ages 20-69 showed $82 \%$ PFS at 2 years (54). In pediatrics, Bv has also been used after HDT/AHCT in ages 16-22. Retrospective analysis showed tolerability of $\mathrm{Bv}$ and $100 \% \mathrm{CR}$ in 5 patients (28).

CAR $T$ products directed at $\mathrm{CD} 30$ are being evaluated for safety and efficacy with early results showing variable responses in relapsed/refractory CD30 positive lymphomas. Various costimulatory domains are being evaluated to improve outcomes including CD28 and CD137. Different lymphodepletion regimens affect efficacy with fludarabine leading to the best outcomes (29, 32, $45,55,56)$. A study of CD30 CAR T cells showed an encouraging ORR of $72 \%$ and CR of $59 \%$ in heavily pretreated patients ages 17 69 (57). Alternatively, CAR T directed at CD123 is under investigation given expression in $50-60 \%$ of Reed-Sternberg cells and the tumor microenvironment $(29,58,59)$.

Other targeted therapies based on the biology and epidemiology of HL include JAK inhibitors, lenalidomide, everolimus, mocetinostat, panobinostat, and vorinostat $(29,58)$. Additionally, preclinical studies are showing the restoration of the typical B cell phenotype to retrieve CD19 expression allows for targeting by CD19 specific agents like blinatumomab or CD19 CAR T cells. Alternatively, CD20 retrieval combined with arsenic trioxide restores CD20 and allows for targeting with anti-CD20 monoclonal antibodies $(29,60)$.

\section{IMPORTANCE OF PATIENT-REPORTED OUTCOMES IN FRONTLINE CARE AND SURVIVORSHIP CARE}

Given the excellent disease outcomes, minimizing acute and late effects of therapy can help determine the best regimens for individual patients. Incorporation of PROs as secondary and exploratory aims in the setting of clinical trials can help inform comparison across studies based on efficacy and patient experience (61). PROs are self-reported using validated questionnaires and can encompass physical, social, and emotional impacts of disease and treatment (62). Incorporation of PRO measurement into cancer care and creation of newer tools have helped drive the field forward $(61,63-66)$.

Retrospectively, Johannsdottir reported $63 \%$ of Norwegian childhood lymphoma survivors treated from 1970-2000 reported psychosocial adverse health outcomes and $97 \%$ reported at least one physical adverse health outcome using the Medical Outcome Study short form-36, CTCAE version 4.0, the Hospital Anxiety and Depression Scale, and the Fatigue Questionnaire. The 
majority of patients underwent combined chemotherapy and RT with a trend toward chemotherapy-only patients reporting better general health than patients undergoing combined modality or RT alone (67).

Berkman described the inclusion of PROs in phase 3 clinical trials in HL including the AYA population between 2007-2020 using the European Organization for Research and Treatment of Cancer Quality of Life Core Questionnaire (QLQ-C30). Four trials (17.4\%) included PROs, but none have yet published the results (62). This is something to look forward to as studies and data mature, and, as suggested by Leblanc, we should expect PRO aims to be published with the primary results of any trial (61). The COG has endorsed and prioritized PRO inclusion in trials, demonstrating the willingness of cooperative groups to both collect and analyze the data (62).

KEYNOTE-087 reported on PROs including reports of health-related quality of life metrics as well as the response rate data showing greater than $70 \%$ of questionnaires were completed. For health-related quality of life scoring using the QLQ-C30 and the EuroQoL Five Dimensions Questionnaire, all three cohorts had similar baseline scores and improvement in both functional and symptom domains after initiation of treatment. Those with partial or complete responses had more improvement in their PRO measures than patients with stable or progressive disease (68).

There are many PRO options that can be incorporated into clinical trials, making comparisons more challenging. Standardization of PROs and comprehensive data collection can provide valuable data to assist in therapeutic decision-making for individual clinicians, individual patients, and cooperative groups planning future clinical trials. With both upfront reporting during clinical trials and follow-up in a survivorship setting, PROs can help provide a meaningful comparison of regimens regarding patient experience during and following therapy.

One example of a gap in care that could be narrowed with PROs is evaluation of psychosocial stressors. Distress in HL has been reported to exceed $30 \%$, and recent work by Troy revealed distress levels were highest during active treatment, related to patient stress and experience of disease and therapy $(65,69)$. Worry and nervousness were also reported as acute psychosocial stressors (3). Addressing unmet needs as reported directly by the patient in a timely manner during therapy and in long-term follow-up can help alleviate some of the burden of the treatment experience, and these experiences may not be identified easily by other means. PROs provide standardized and validated ways to collect this data which is potentially actionable at the patient level but also by collaborative groups to better understand complications of treatment that may warrant further investigation.

The patient report of physical symptoms is also important with common symptoms being fatigue, nausea, and pain (3). Longterm health effects of HL therapy have traditionally included pulmonary fibrosis, pneumonitis, heart disease, thyroid dysfunction, chronic fatigue, neurocognitive effects, osteoporosis, and sexual dysfunction (70). Tracking of and response to patient report of these symptoms and experiences will continue to grow as a critical component of clinical trials and optimization of care.
This is particularly important with regimens reducing cytotoxic chemotherapy and RT and incorporating newer agents.

With newer agents, toxicity monitoring is essential, particularly in the pediatric population. Short and long-term toxicity may differ between adult and pediatric patients. ICIs have different toxicities than traditional cytotoxic chemotherapies including autoimmune hepatitis, thyroid dysfunction, pneumonitis, colitis, rashes, fatigue, infusion reactions, pyrexia, and more rarely neurologic, renal, ocular, and pancreatic toxicity (71). Given this diverse set of toxicities, incorporation of PROs and structured follow-up of survivors can identify the prevalence and severity of adverse effects in the AYA and pediatric patient as these agents are increasingly being used.

\section{DISCUSSION}

Despite many advances, there remains lack of consensus regarding the best management of $\mathrm{HL}$, and priorities differ for which strategies to evaluate next in clinical trials. Better understanding the patient experience and outcomes through collaboration, clinical trials, and the use of PROs could be an important step forward to achieve the best outcomes and therapy options.

Following patients over the last several decades allowed us to recognize the long-term health effects of curative therapeutic approaches, and subsequently long-term follow-up is essential to avoid trading one toxicity for another. Harmonization efforts for supportive care and long-term follow-up recommendations are underway and will be beneficial to provide therapy-specific and risk-adapted monitoring for toxicities and effects of therapy $(2,6$, 70, 72-74). Ehrhardt makes a compelling argument to assess and consider the risk of late toxicities, converting this risk assessment into actionable data for the choice of upfront therapy (6).

Moving forward, considering toxicities in clinical decisionmaking, continuing to evaluate new treatments through collaborative clinical trials, and formalizing assessment of PROs can help achieve the goals to reduce toxicity and maintain high rates of cure. Continued collaborations can help standardize risk assessment, data collection, and toxicity reporting so trials can more easily be compared. This data will be invaluable when choosing a treatment for an individual patient.

\section{DATA AVAILABILITY STATEMENT}

The original contributions presented in the study are included in the article/supplementary material. Further inquiries can be directed to the corresponding author.

\section{AUTHOR CONTRIBUTIONS}

CS and DF conceptualized, drafted, reviewed, and approved the final manuscript. 


\section{REFERENCES}

1. American Cancer Society. Cancer Facts \& Figures 2020. Atlanta: American Cancer Society (2020). Available at: http://www.cancer.org/acs/groups/ content/@nho/documents/document/caff2007pwsecuredpdf.pdf.

2. Flerlage JE, Metzger ML, Bhakta N. The Management of Hodgkin Lymphoma in Adolescents and Young Adults: Burden of Disease or Burden of Choice? Blood (2018) 132(4):376-84. doi: 10.1182/blood-2018-01-778548

3. Kahn JM, Kelly KM. Adolescent and Young Adult Hodgkin Lymphoma: Raising the Bar Through Collaborative Science and Multidisciplinary Care. Pediatr Blood Cancer (2018) 65(7):1-19. doi: 10.1002/pbc.27033

4. Ries L, Smith M, Gurney J, Linet M, Tamra T, Young JL, et al. Cancer Incidence and Survival Among Children and Adolescents: United States SEER Program 1975-1995 (1999). Bethesda, MD: National Cancer Institute, SEER Program. NIH Pub. No. 9904649. Available at: https://seer.cancer.gov/ archive/publications/childhood/ (Accessed January 5, 2022).

5. Howlader N, Noone A, Krapcho M, Miller D, Brest A, Yu M, et al. Seer Cancer Statistics Review, 1975-2018. Bethesda, MD: National Cancer Institute. Available at: https://seer.cancer.gov/csr/1975_2018/ (Accessed January 5, 2022).

6. Ehrhardt MJ, Flerlage JE, Armenian SH, Castellino SM, Hodgson DC, Hudson MM. Integration of Pediatric Hodgkin Lymphoma Treatment and Late Effects Guidelines: Seeing the Forest Beyond the Trees. JNCCN J Natl Compr Cancer Netw (2021) 19(6):755-64. doi: 10.6004/jnccn.2021.7042

7. Daw S, Hasenclever D, Mascarin M, Fernández-Teijeiro A, Balwierz W, Beishuizen A, et al. Risk and Response Adapted Treatment Guidelines for Managing First Relapsed and Refractory Classical Hodgkin Lymphoma in Children and Young People. Recommendations From the Euronet Pediatric Hodgkin Lymphoma Group. HemaSphere (2020) 4(1):e329. doi: 10.1097/ HS9.0000000000000329

8. Zhou R, Ng A, Constine LS, Stovall M, Armstrong GT, Neglia JP, et al. A Comparative Evaluation of Normal Tissue Doses for Patients Receiving Radiation Therapy for Hodgkin Lymphoma on the Childhood Cancer Survivor Study and Recent Children's Oncology Group Trials. Int J Radiat Oncol Biol Phys (2016) 95(2):707-11. doi: 10.1016/J.IJROBP.2016.01.053

9. Friedman DL, Chen L, Wolden S, Buxton A, McCarten K, FitzGerald TJ, et al. Dose-Intensive Response-Based Chemotherapy and Radiation Therapy for Children and Adolescents With Newly Diagnosed Intermediate-Risk Hodgkin Lymphoma: A Report From the Children's Oncology Group Study AHOD0031. J Clin Oncol (2014) 32(32):3651-8. doi: 10.1200/ JCO.2013.52.5410

10. Keller FG, Castellino SM, Chen L, Pei Q, Voss SD, McCarten KM, et al. Results of the AHOD0431 Trial of Response Adapted Therapy and a Salvage Strategy for Limited Stage, Classical Hodgkin Lymphoma: A Report From the Children's Oncology Group. Cancer (2018) 124(15):3210-9. doi: 10.1002/ CNCR.31519

11. Kelly KM, Cole PD, Pei Q, Bush R, Roberts KB, Hodgson DC, et al. ResponseAdapted Therapy for the Treatment of Children With Newly Diagnosed High Risk Hodgkin Lymphoma (AHOD0831): A Report From the Children's Oncology Group. Br J Haematol (2019) 187(1):39-48. doi: 10.1111/bjh.16014

12. Mauz-Körholz C, Landman-Parker J, Balwierz W, Ammann RA, Anderson RA, Attarbaschi A, et al. Response-Adapted Omission of Radiotherapy and Comparison of Consolidation Chemotherapy in Children and Adolescents With Intermediate-Stage and Advanced-Stage Classical Hodgkin Lymphoma (Euronet-PHL-C1): A Titration Study With an Open-Label, Embedded, Multinational, non-Inferiority, Randomised Controlled Trial. Lancet Oncol (2022) 23(1):125-37. doi: 10.1016/S1470-2045(21)00470-8

13. Mauz-Körholz C, Metzger ML, Kelly KM, Schwartz CL, Castellanos M, Dieckmann K, et al. Pediatric Hodgkin Lymphoma. J Clin Oncol (2015) 33 (27):2975-85. doi: 10.1200/JCO.2014.59.4853

14. Gordon LI, Hong F, Fisher RI, Bartlett NL, Connors JM, Gascoyne RD, et al. Randomized Phase III Trial of ABVD Versus Stanford V With or Without Radiation Therapy in Locally Extensive and Advanced-Stage Hodgkin Lymphoma: An Intergroup Study Coordinated by the Eastern Cooperative Oncology Group (E2496). J Clin Oncol (2013) 31(6):684. doi: 10.1200/ JCO.2012.43.4803

15. Stephens DM, Li H, Schöder H, Straus DJ, Moskowitz CH, LeBlanc M, et al. Five-Year Follow-Up of SWOG S0816: Limitations and Values of a PET-
Adapted Approach With Stage III/IV Hodgkin Lymphoma. Blood (2019) 134 (15):1238-46. doi: 10.1182/blood.2019000719

16. Press OW, Li H, Schöder H, Straus DJ, Moskowitz CH, LeBlanc M, et al. US Intergroup Trial of Response-Adapted Therapy for Stage III to IV Hodgkin Lymphoma Using Early Interim Fluorodeoxyglucose-Positron Emission Tomography Imaging: Southwest Oncology Group S0816. J Clin Oncol (2016) 34(17):2020-7. doi: 10.1200/JCO.2015.63.1119

17. Gopal AK, Chen R, Smith SE, Ansell SM, Rosenblatt JD, Savage KJ, et al. Durable Remissions in a Pivotal Phase 2 Study of Brentuximab Vedotin in Relapsed or Refractory Hodgkin Lymphoma. Blood (2015) 125(8):1236-43. doi: 10.1182/BLOOD-2014-08-595801

18. Chen R, Gopal AK, Smith SE, Ansell SM, Rosenblatt JD, Savage KJ, et al. FiveYear Survival and Durability Results of Brentuximab Vedotin in Patients With Relapsed or Refractory Hodgkin Lymphoma. Blood (2016) 128(12):1562-6. doi: 10.1182/BLOOD-2016-02-699850

19. Cole PD, McCarten KM, Pei Q, Spira M, Metzger ML, Drachtman RA, et al. Brentuximab Vedotin With Gemcitabine for Paediatric and Young Adult Patients With Relapsed or Refractory Hodgkin's Lymphoma (AHOD1221): A Children's Oncology Group, Multicentre Single-Arm, Phase 1-2 Trial. Lancet Oncol (2018) 19(9):1229-38. doi: 10.1016/S1470-2045(18)30426-1

20. LaCasce AS, Gregory Bociek R, Sawas A, Caimi P, Agura E, Matous J, et al. Brentuximab Vedotin Plus Bendamustine: A Highly Active First Salvage Regimen for Relapsed or Refractory Hodgkin Lymphoma. Blood (2018) 132 (1):40-8. doi: 10.1182/BLOOD-2017-11-815183

21. Broccoli A, Argnani L, Botto B, Corradini P, Pinto A, Re A, et al. First Salvage Treatment With Bendamustine and Brentuximab Vedotin in Hodgkin Lymphoma: A Phase 2 Study of the Fondazione Italiana Linfomi. Blood Cancer J (2019) 9(12):1-8. doi: 10.1038/s41408-019-0265-x

22. Forlenza CJ, Gulati N, Mauguen A, Absalon MJ, Castellino SM, Franklin A, et al. Combination Brentuximab Vedotin and Bendamustine for Pediatric Patients With Relapsed/Refractory Hodgkin Lymphoma. Blood Adv (2021) 5 (24):5519-24. doi: 10.1182/BLOODADVANCES.2021005268

23. O'Connor OA, Lue JK, Sawas A, Amengual JE, Deng C, Kalac M, et al. Brentuximab Vedotin Plus Bendamustine in Relapsed or Refractory Hodgkin's Lymphoma: An International, Multicentre, Single-Arm, Phase 12 Trial. Lancet Oncol (2018) 19(2):257-66. doi: 10.1016/S1470-2045(17) 30912-9

24. Metzger ML, Link MP, Billett AL, Flerlage J, Lucas JT, Mandrell BN, et al. Excellent Outcome for Pediatric Patients With High-Risk Hodgkin Lymphoma Treated With Brentuximab Vedotin and Risk-Adapted Residual Node Radiation. J Clin Oncol (2021) 39(20):2276-83. doi: 10.1200/ jco. 20.03286

25. Brentuximab Vedotin and Combination Chemotherapy in Treating Children and Young Adults With Stage IIB or Stage IIIB-IVB Hodgkin Lymphoma. Clinicaltrials.Gov Identifier: NCT02166463. Available at: https://www. clinicaltrials.gov/ct2/show/NCT02166463 (Accessed January 2, 2022).

26. Connors JM, Jurczak W, Straus DJ, Ansell SM, Kim WS, Gallamini A, et al. Brentuximab Vedotin With Chemotherapy for Stage III or IV Hodgkin's Lymphoma. N Engl J Med (2018) 378(4):331-44. doi: 10.1056/ NEJMoa1708984

27. Straus DJ, Długosz-Danecka M, Alekseev S, Illés Á, Picardi M, Lech-Maranda E, et al. Brentuximab Vedotin With Chemotherapy for Stage III/IV Classical Hodgkin Lymphoma: 3-Year Update of the ECHELON-1 Study. Blood (2020) 135(10):735-42. doi: 10.1182/BLOOD.2019003127

28. Flerlage JE, von Buttlar X, Krasin M, Triplett B, Kaste SC, Metzger ML. Brentuximab Vedotin as Consolidation After Hematopoietic Cell Transplant for Relapsed Hodgkin Lymphoma in Pediatric Patients. Pediatr Blood Cancer (2019) 66(12):1-2. doi: 10.1002/pbc.27962

29. Mottok A, Steidl C. Biology of Classical Hodgkin Lymphoma: Implications for Prognosis and Novel Therapies. Blood (2018) 131(15):1654-65. doi: 10.1182/ blood-2017-09-772632

30. Chen R, Zinzani PL, Lee HJ, Armand P, Johnson NA, Brice P, et al. Pembrolizumab in Relapsed or Refractory Hodgkin Lymphoma: 2-Year Follow-Up of KEYNOTE-087. Blood (2019) 134(14):1144-53. doi: 10.1182/ blood.2019000324

31. Ramchandren R, Domingo-Domènech $\mathrm{E}$, Rueda $\mathrm{A}$, Trněný $\mathrm{M}$, Feldman TA, Lee HJ, et al. Nivolumab for Newly Diagnosed Advanced-Stage Classic 
Hodgkin Lymphoma: Safety and Efficacy in the Phase II Checkmate 205 Study. J Clin Oncol (2019) 37(23):1997-2007. doi: 10.1200/JCO.19.00315

32. Matsuki E, Younes A. Checkpoint Inhibitors and Other Immune Therapies for Hodgkin and Non-Hodgkin Lymphoma. Curr Treat Options Oncol (2016) 17(6):31. doi: 10.1007/S11864-016-0401-9

33. Diefenbach CS, Hong F, Ambinder RF, Cohen JB, Robertson MJ, David KA, et al. Ipilimumab, Nivolumab, and Brentuximab Vedotin Combination Therapies in Patients With Relapsed or Refractory Hodgkin Lymphoma: Phase 1 Results of an Open-Label, Multicentre, Phase 1/2 Trial. Artic Lancet Haematol (2020) 7:660-70. doi: 10.1016/S2352-3026(20)30221-0

34. Armand P, Engert A, Younes A, Fanale M, Santoro A, Zinzani PL, et al. Nivolumab for Relapsed/Refractory Classic Hodgkin Lymphoma After Failure of Autologous Hematopoietic Cell Transplantation: Extended Follow-Up of the Multicohort Single-Arm Phase II Checkmate 205 Trial. J Clin Oncol (2018) 36(14):1428-39. doi: 10.1200/JCO.2017.76.0793

35. Ansell SM, Bröckelmann PJ, Keudell G, Lee HJ, Santoro A, Zinzani PL, et al. Nivolumab for Relapsed or Refractory (R/R) Classical Hodgkin Lymphoma (CHL) After Autologous Transplantation: 5-Year Overall Survival From the Phase 2 Checkmate 205 Study. Hematol Oncol (2021) 39(S2):122-5. doi: 10.1002/HON.74_2879

36. Younes A, Santoro A, Shipp M, Zinzani PL, Timmerman JM, Ansell S, et al. Nivolumab for Classical Hodgkin's Lymphoma After Failure of Both Autologous Stem-Cell Transplantation and Brentuximab Vedotin: A Multicentre, Multicohort, Single-Arm Phase 2 Trial. Lancet Oncol (2016) 17 (9):1283-94. doi: 10.1016/S1470-2045(16)30167-X

37. Chen R, Zinzani PL, Fanale MA, Armand P, Johnson NA, Brice P, et al. Phase II Study of the Efficacy and Safety of Pembrolizumab for Relapsed/Refractory Classic Hodgkin Lymphoma. J Clin Oncol (2017) 35(19):2125-32. doi: 10.1200/JCO.2016.72.1316

38. Brentuximab Vedotin and Nivolumab With or Without Ipilimumab in Treating Patients With Relapsed or Refractory Hodgkin Lymphoma. Clinicaltrials.Gov Identifier: NCT01896999. Available at: https://clinicaltrials. gov/ct2/show/NCT01896999 (Accessed January 6, 2022).

39. Voorhees TJ, Beaven AW. Therapeutic Updates for Relapsed and Refractory Classical Hodgkin Lymphoma. Cancers (Basel) (2020) 12(10):2887. doi: $10.3390 /$ cancers 12102887

40. Diefenbach C, Hong F, Ambinder RF, Cohen JB, Robertson M, David KA, et al. A Phase I Study With an Expansion Cohort of the Combinations of Ipilimumab, Nivolumab and Brentuximab Vedotin in Patients With Relapsed/Refractory Hodgkin Lymphoma: A Trial of the ECOG-ACRIN Research Group (E4412: Arms G-I). Blood (2018) 132(Supplement 1):6799. doi: 10.1182/BLOOD-2018-99-115390

41. Davis KL, Fox E, Merchant MS, Reid JM, Kudgus RA, Liu X, et al. Nivolumab in Children and Young Adults With Relapsed or Refractory Solid Tumours or Lymphoma (ADVL1412): A Multicentre, Open-Label, Single-Arm, Phase 1-2 Trial. Lancet Oncol (2020) 21(4):541-50. doi: 10.1016/S1470-2045(20)30023-1

42. Safety and Efficacy of Pembrolizumab (MK-3475) in Children and Young Adults With Classical Hodgkin Lymphoma (MK-3475-667/KEYNOTE-667). Clinicaltrials.Gov Identifier: NCT03407144. Available at: https://clinicaltrials. gov/ct2/show/NCT03407144 (Accessed January 6, 2022).

43. Immunotherapy (Nivolumab or Brentuximab Vedotin) Plus Combination Chemotherapy in Treating Patients With Newly Diagnosed Stage III-IV Classic Hodgkin Lymphoma. Clinicaltrials.Gov Identifier: NCT03907488. Available at: https://www.clinicaltrials.gov/ct2/show/NCT03907488 (Accessed January 6, 2022).

44. Moskowitz AJ, Herrera AF, Beaven AW. Relapsed and Refractory Classical Hodgkin Lymphoma: Keeping Pace With Novel Agents and New Options for Salvage Therapy. Am Soc Clin Oncol Educ B (2019) 39):477-86. doi: 10.1200/ edbk_238799

45. Othman T, Herrera A, Mei M. Emerging Therapies in Relapsed and Refractory Hodgkin Lymphoma: What Comes Next After Brentuximab Vedotin and PD-1 Inhibition? Curr Hematol Malig Rep (2021) 16(1):1-7. doi: 10.1007/S11899-020-00603-3

46. NCCN Guidelines, Hoppe RT, Advani RH, Ai WZ. NCCN Clinical Practice Guidelines in Oncology: Hodgkin Lymphoma. Version 1.2022. Available at: www.nccn.org (Accessed January 4, 2022).

47. Guidelines NCCN, Flerlage JE, SM H, Armenian S. NCCN Clinical Practice Guidelines in Oncology: Pediatric Hodgkin Lymphoma. Version 3.2021. Available at: www.nccn.org (Accessed January 4, 2022).
48. Harker-Murray PD, Drachtman RA, Hodgson DC, Chauvenet AR, Kelly KM, Cole PD. Stratification of Treatment Intensity in Relapsed Pediatric Hodgkin Lymphoma. Pediatr Blood Cancer (2014) 61(4):579-86. doi: 10.1002/ pbc. 24851

49. Moskowitz AJ, Shah G, Schöder H, Ganesan N, Drill E, Hancock H, et al. Phase II Trial of Pembrolizumab Plus Gemcitabine, Vinorelbine, and Liposomal Doxorubicin as Second-Line Therapy for Relapsed or Refractory Classical Hodgkin Lymphoma. J Clin Oncol (2021) 39(28):3109-17. doi: $10.1200 /$ jco. 21.01056

50. Harker-Murray P, Leblanc T, Mascarin M, Mauz-Körholz C, Michel G, Cooper S, et al. Response-Adapted Therapy With Nivolumab and Brentuximab Vedotin (BV), Followed by BV and Bendamustine for Suboptimal Response, in Children, Adolescents, and Young Adults With Standard-Risk Relapsed/Refractory Classical Hodgkin Lymphoma. Blood (2018) 132(Supplement 1):927. doi: 10.1182/BLOOD-2018-99-111279

51. Cole PD, Mauz-Körholz C, Mascarin M, Michel G, Cooper S, Beishuizen A, et al. Nivolumab and Brentuximab Vedotin (BV)-Based, Response-Adapted Treatment in Children, Adolescents, and Young Adults (CAYA) With Standard-Risk Relapsed/Refractory Classical Hodgkin Lymphoma (R/R Chl): Primary Analysis. J Clin Oncol (2020) 38(15_suppl):8013-3. doi: 10.1200/JCO.2020.38.15_SUPPL.8013

52. Moskowitz CH, Nademanee A, Masszi T, Agura E, Holowiecki J, Abidi MH, et al. Brentuximab Vedotin as Consolidation Therapy After Autologous StemCell Transplantation in Patients With Hodgkin's Lymphoma at Risk of Relapse or Progression (AETHERA): A Randomised, Double-Blind, Placebo-Controlled, Phase 3 Trial. Lancet (2015) 385(9980):1853-62. doi: 10.1016/S0140-6736(15)60165-9

53. Moskowitz CH, Walewski J, Nademanee A, Masszi T, Agura E, Holowiecki J, et al. Five-Year PFS From the AETHERA Trial of Brentuximab Vedotin for Hodgkin Lymphoma at High Risk of Progression or Relapse. Blood (2018) 132 (25):2639-42. doi: 10.1182/blood-2018-07-861641

54. Armand P, Chen YB, Redd RA, Joyce RM, Bsat J, Jeter E, et al. PD-1 Blockade With Pembrolizumab for Classical Hodgkin Lymphoma After Autologous Stem Cell Transplantation. Blood (2019) 134(1):22-9. doi: 10.1182/ BLOOD.2019000215

55. Brice P, de Kerviler E, Friedberg JW. Classical Hodgkin Lymphoma. Lancet (2021) 398(10310):1518-27. doi: 10.1016/S0140-6736(20)32207-8

56. Dahi PB, Moskowitz CH, Giralt SA, Lazarus HM. Novel Agents Positively Impact Chemotherapy and Transplantation in Hodgkin Lymphoma. Expert Rev Hematol (2019) 12(4):255-64. doi: 10.1080/17474086.2019.1593135

57. Ramos CA, Grover NS, Beaven AW, Lulla PD, Wu MF, Ivanova A, et al. AntiCD30 CAR-T Cell Therapy in Relapsed and Refractory Hodgkin Lymphoma. J Clin Oncol (2020) 38(32):3794-804. doi: 10.1200/JCO.20.01342

58. Mehta-Shah N, Bartlett NL. Management of Relapsed/Refractory Classical Hodgkin Lymphoma in Transplant-Ineligible Patients. Blood (2018) 131 (15):1698-703. doi: 10.1182/blood-2017-09-772681

59. Ruella M, Klichinsky M, Kenderian SS, Shestova O, Ziober A, Kraft DO, et al. Overcoming the Immunosuppressive Tumor Microenvironment of Hodgkin Lymphoma Using Chimeric Antigen Receptor T Cells. Cancer Discov (2017) 7 (10):1154-67. doi: 10.1158/2159-8290.CD-16-0850

60. Du J, Neuenschwander M, Yu Y, Däbritz JH, Neuendorff NR, Schleich K, et al. Pharmacological Restoration and Therapeutic Targeting of the B-Cell Phenotype in Classical Hodgkin Lymphoma. Blood (2017) 129(1):71-81. doi: 10.1182/BLOOD-2016-02-700773

61. Leblanc TW. Experts on Their Own Experiences: The Rise of PatientReported Outcomes in Oncology Drug Trials. Leuk Lymphoma (2019) 60 (11):2604-5. doi: 10.1080/10428194.2019.1632446

62. Berkman AM, Murphy KM, Siembida EJ, Lau N, Geng Y, Parsons SK, et al. Inclusion of Patient-Reported Outcomes in Adolescent and Young Adult Phase III Therapeutic Trials: An Analysis of Cancer Clinical Trials Registered on Clinicaltrials.Gov. Value Health (2021) 24(12):1820-7. doi: 10.1016/ j.jval.2021.06.012

63. Basch E. Patient-Reported Outcomes - Harnessing Patients' Voices to Improve Clinical Care. N Engl J Med (2017) 376(2):105-8. doi: 10.1056/ nejmp1611252

64. Basch E, Barbera L, Kerrigan CL, Velikova G. Implementation of PatientReported Outcomes in Routine Medical Care. Am Soc Clin Oncol Educ B (2018) 38):122-34. doi: 10.1200/edbk_200383 
65. Tarnasky AM, Troy JD, Leblanc TW. The Patient Experience of ABVD Treatment in Hodgkin Lymphoma : A Retrospective Cohort Study of PatientReported Distress. Support Care Cancer (2021) 29(9):4987-96. doi: 10.1007/ s00520-021-06044-9

66. LeBlanc TW, Abernethy AP. Patient-Reported Outcomes in Cancer Care Hearing the Patient Voice at Greater Volume. Nat Rev Clin Oncol (2017) 14 (12):763-72. doi: 10.1038/nrclinonc.2017.153

67. Johannsdottir IMR, Hamre H, Fossa SD, Loge JH, Drolsum L, Lund MB, et al. Adverse Health Outcomes and Associations With Self-Reported General Health in Childhood Lymphoma Survivors. J Adolesc Young Adult Oncol (2017) 6(3):470-6. doi: 10.1089/jayao.2017.0018

68. von Tresckow B, Fanale M, Ardeshna KM, Chen R, Meissner J, Morschhauser F, et al. Patient-Reported Outcomes in KEYNOTE-087, a Phase 2 Study of Pembrolizumab in Patients With Classical Hodgkin Lymphoma. Leuk Lymphoma (2019) 60(11):2705-11. doi: 10.1080/10428194.2019.1602262

69. Troy JD, Locke SC, Samsa GP, Feliciano J, Richhariya A, Leblanc TW. PatientReported Distress in Hodgkin Lymphoma Across the Survivorship Continuum. Support Care Cancer (2019) 27:2453-62. doi: 10.1007/s00520018-4523-4

70. Frick MA, Vachani CC, Hampshire MK, Bach C, Arnold-Korzeniowski K, Metz JM, et al. Patient-Reported Survivorship Care Practices and Late Effects After Treatment of Hodgkin and non-Hodgkin Lymphoma. JCO Clin Cancer Inform (2018) 2):1-10. doi: 10.1200/cci.18.00015

71. Naidoo J, Page DB, Li BT, Connell LC, Schindler K, Lacouture ME, et al. Toxicities of the Anti-PD-1 and Anti-PD-L1 Immune Checkpoint Antibodies. Ann Oncol Off J Eur Soc Med Oncol (2015) 26(12):2375-91. doi: 10.1093/ ANNONC/MDV383

72. Landier W, Bhatia S, Eshelman DA, Forte KJ, Sweeney T, Hester AL, et al. Development of Risk-Based Guidelines for Pediatric Cancer Survivors: The Children's Oncology Group Long-Term Follow-Up Guidelines From the
Children's Oncology Group Late Effects Committee and Nursing Discipline. J Clin Oncol (2004) 22(24):4979-90. doi: 10.1200/JCO.2004.11.032

73. Kremer LC, Mulder RL, Oeffinger KC, Bhatia S, Landier W, Levitt G, et al. A Worldwide Collaboration to Harmonize Guidelines for the Long-Term Follow-Up of Childhood and Young Adult Cancer Survivors: A Report From the International Late Effects of Childhood Cancer Guideline Harmonization Group. Pediatr Blood Cancer (2013) 60(4):543-9. doi: 10.1002/PBC.24445

74. Bhatia S, Armenian SH, Armstrong GT, van Dulmen-den Broeder E, Hawkins MM, Kremer LC, et al. Collaborative Research in Childhood Cancer Survivorship: The Current Landscape. J Clin Oncol (2015) 33(27):3055. doi: 10.1200/JCO.2014.59.8052

Conflict of Interest: The authors declare that the research was conducted in the absence of any commercial or financial relationships that could be construed as a potential conflict of interest.

Publisher's Note: All claims expressed in this article are solely those of the authors and do not necessarily represent those of their affiliated organizations, or those of the publisher, the editors and the reviewers. Any product that may be evaluated in this article, or claim that may be made by its manufacturer, is not guaranteed or endorsed by the publisher.

Copyright (C) 2022 Smith and Friedman. This is an open-access article distributed under the terms of the Creative Commons Attribution License (CC BY). The use, distribution or reproduction in other forums is permitted, provided the original author(s) and the copyright owner(s) are credited and that the original publication in this journal is cited, in accordance with accepted academic practice. No use, distribution or reproduction is permitted which does not comply with these terms. 